\title{
El diseño curricular por competencias en educación médica: impacto en la formación profesional.
}

\author{
Curricular design by competences in medical education: \\ impact on the professional training
}

*Red iberoamericana para la formación continua de los docentes de áreas biomédicas.

"Formas de enseñar - formas de aprender en la Universidad" (FE-FAU)

José-Vicente Lafuente ${ }^{1}$, Jesús F. Escanero ${ }^{2}$, Jose $\mathrm{M}^{\mathrm{a}} \mathrm{Manso}^{3}$, Sergio Mora ${ }^{4}$, Teresa Miranda ${ }^{4}$, Manuel Castillo $^{4}$, Gabriela Díaz-Veliz ${ }^{4}$, Pascual Gargiulo ${ }^{5}$, Ricardo Bianchi ${ }^{5}$, Dorian Gorena ${ }^{6}$ y José Mayora ${ }^{6}$

${ }^{1}$ (U. del País Vasco-Euskal Herriko U., Leioa, España); ${ }^{2}$ (U. de Zaragoza, Zaragoza, España); ${ }^{3}$ (U. de Valladolid, Valladolid, España); ${ }^{4}$ (U. de Chile, Santiago de Chile, Chile); ${ }^{5}$ (U. Ncal. de Cuyo, Mendoza, Argentina); ${ }^{6}$ (U. Andina Simón Bolivar y U. Mayor de S. Fco. de Chuquisaca, Sucre, Bolivia).

La universidad está en un proceso de refundación con profundos cambios epistemológicos. La decisión de la UE de desarrollar una "economía basada en el conocimiento" requiere el establecimiento de objetivos educativos comunes que desarrolla el proceso de Bolonia, herramienta clave de la construcción del Espacio Europeo de Educación Superior. En este contexto la universidad, tradicionalmente regida por el paradigma conductista, constata que las competencias definen los perfiles profesionales. Este concepto esta vinculado al diseño de los nuevos curricula.

El diseño curricular es una selección cultural, un ejercicio de "apreciar y excluir". El currículum debe responder a: ¿Cuál es el conocimiento válido?, ¿Cómo se adquieren los conocimientos, habilidades y actitudes? y ¿Cómo evaluar el logro de las competencias?

Las competencias son una combinación de conocimientos, habilidades y actitudes conducentes

Correspondencia:

J.V. Lafuente

Depto. Neurociencias

Universidad del País Vasco- Euskal Herriko Unibertsitatea

Apdo. 699

48080-Bilbao

e-mail: josevicente.lafuente@ehu.es al desempeño adecuado y oportuno de una tarea en el campo de las ciencias de la salud. Las hay básicas, con las que cada uno construimos nuestro aprendizaje, personales, las que nos permiten actuar responsablemente, y profesionales, las que garantizan el cumplimiento del ejercicio profesional.

Los modelos por competencias profesionales integradas buscan generar procesos formativos de alta calidad. Ello implica promover acciones que supongan modificaciones reales en la práctica docente con un acercamiento dinámico a la realidad del mundo circundante. Ello sólo puede ser acometido si el estudiante asume un papel activo en su aprendizaje. En esta línea se insta a órganos de gobierno, sociedades profesionales y foros independientes de opinión a colaborar en la refundación de la universidad en los comienzos del siglo XXI.

Palabras clave: Competencia, Diseño Curricular, Proceso de Bolonia, Espacio Europeo de Enseñanza Superior, Refundación de la Universidad

The University is undergoing a process of refoundation with far-reaching epistemological changes.

The EU's decision of developing a "knowledge based economy" need to establish common educational 
objectives, which are implemented by the Bologna process, a key tool in the construction of the European Space of High Education.

In this context, the University, traditionally leaded by the behaviourist paradigm, found the competence concept impregnating all professional profiles.

This concept is linked to the new curricular design. Curricular design is a cultural selection, an exercise in "appreciate and exclude". The curriculum must respond to some questions: "What knowledge is valid? How do we acquire knowledge, skills and attitudes? And how do we evaluate the achievement of competences? Competences are a combination of knowledge, skills and attitudes leading to perform an adequate and precise professional role in health sciences. They can be basics, to build our learning; personals, which enable us to act responsibly; and professionals, those that assure the accomplishment of professional tasks. Models based on integrated professional competences seek to produce high quality educational processes. This implies to promote actions that result in changes in teaching with a dynamic approach towards the real context. It can only be carried out if the student takes an active role in his/her learning.

For this scope, all stakeholders (government, professional societies and independent opinion organizations) are encouraged to collaborate in the refoundation of the University today at the beginning of the 21 st Century.

Key words: Competence, Curricular Design, Bologna Process, European Space of High Education, University Refoundation

Una serie de hechos están colocando a la Universidad y a los universitarios en una coyuntura crítica; a una y a otros les está exigiendo rápidas adaptaciones y profundos cambios en sus planteamientos y quehacer cotidiano, pero no todos están preparados para ello. Esta situación ha llevado a muchos a asumir que nos encontramos en un "cambio de época" más que en una época de cambios. Esta formulación ayuda a entender la magnitud del proceso en que se halla inmersa la Universidad, no se trata sólo de una redefinición ${ }^{1}$, se apunta decididamente a un cambio epistemológico que conducirá a una refundación de la misma.

\section{SOBRE EL CONTEXTO SOCIO-POLÍTICO}

La Unión Europea (UE) es hoy una realidad política y económica con avances visibles y logros relevantes en el camino de la construcción de un estado transnacional europeo ${ }^{2}$. A finales de los noventa se iniciaron dos procesos de gran importancia para el futuro de los sistemas educativos europeos: Uno es el establecimiento de unos objetivos educativos comunes (objetivos de Lisboa), y otro es el conocido como proceso Bolonia.

La voluntad de la UE de convertirse en una economía basada en el conocimiento, inspiró los denominados objetivos de Lisboa. La UE se ha propuesto desarrollar plenamente su capital humano, lo que se ha traducido en una serie de objetivos educativos que deberían alcanzarse para el año 2010. Estas metas están orientadas a mejorar la calidad y la eficacia de la educación, facilitar el acceso a la misma y abrir los sistemas educativos a un mundo más amplio. El proceso Bolonia puede ser considerado como el producto de un conjunto de decisiones políticas sobre la construcción de un Espacio Europeo de Educación Superior (EEES) ${ }^{3}$.

Los cambios indicados, que tienen en los objetivos de Lisboa y en la Declaración de Bolonia (1999) sus referentes, han cristalizado en una serie de declaraciones políticas sucesivas. En la última reunión celebrada en la ciudad de Bergen (Noruega), en 2005, fueron ya 45 los países adscritos a este espacio y otros muchos, especialmente iberoamericanos, los que asistieron en calidad de invitados. Para tener una idea de la evolución del proceso sirva recordar que la Declaración de La Sorbona (1998) la firmaron cuatro países: Alemania, Francia, Italia y Reino Unido.

\section{SOBRE LA UNIVERSIDAD}

La Universidad actual, heredera de la Universidad de los setenta, se encuentra expectante y preocupada por el futuro. En aquella Universidad, la formación se entendía separada de las actividades de producción y servicios y la enseñanza se centraba en la adquisición de conocimientos disciplinares especializados. $\mathrm{Su}$ paradigma era el conductista y se desconocía el significado del término competencia (tanto profesional, como intelectual o personal $)^{4}$. Se trataba de una Universidad encerrada en sí misma, que vivía en una auténtica torre de cristal, alejada de las demandas y necesidades de la Sociedad en que estaba insertada. En la década siguiente (la de los ochenta), la crisis 
económica y la fuerte competencia comercial hicieron que el planteamiento anterior quedase obsoleto $\mathrm{y}$ se legislaron reformas universitarias en diferentes países. Se insinuaba la necesidad de volver la mirada hacia los recursos humanos que las empresas necesitaban para subsistir en dicho entorno de competitividad. Esto requería de habilidades y competencias más allá de los conocimientos académicos y se reclamaba un cambio en el papel de la formación, que debía ser mucho más práctica. La realidad de la Universidad se transformó desde la perspectiva curricular con la incorporación de conceptos como planificación-programación (basados en objetivos), la introducción de ratios teoría-práctica (favoreciendo esta última) y la incorporación de formas concretas de evaluación. Se continuó con el mismo paradigma y la realidad ha sido decepcionante, ya que en una pirueta descomunal las prácticas se trasmutaron en teoría, incrementando en definitiva, la carga de conocimientos a retener. En la última década del siglo pasado cuando ya se estaba legislando a un ritmo inusitado sobre el EEES todavía hubo facultades que se embarcaron en estos cambios, introduciendo más asignaturas y, en consecuencia, más contenidos.

De la Universidad de los setenta, encerrada en sí misma, a la "diseñada" en Bolonia basada en la Sociedad del Conocimiento y fundamentada en la emergente sociedad post-industrial, con una clara vocación de inserción y utilidad social, va un largo recorrido, donde las competencias que deben definir el perfil profesional del egresado jalonan su trayecto ${ }^{5}$.

\section{SOBRE LA FORMACIÓN MÉDICA}

La evolución de la educación médica, desde 1950 hasta la actualidad, permite diferenciar tres periodos distintos ${ }^{6}$ :

a. Un primer período de planificación por objetivos, inspirado por los métodos de gestión que se han impuesto a partir de los años cincuenta.

b. Un segundo período, a partir de los años setenta, caracterizado por la búsqueda de la pertinencia con el fin de conseguir un médico en concordancia con la situación sanitaria local, con la motivación para identificar las necesidades cualitativas y cuantitativas.

c. Un tercer período, a partir de los noventa, caracterizado por la búsqueda del impacto de la educación médica sobre la salud de la población, lo que implica un proceso tendente a transformar la Facultad de Medicina en un "partenaire" explícito del desarrollo sanitario.
Estos tres períodos se solapan en la actualidad poniendo sucesivamente énfasis en la gestión, la pertinencia y el impacto sobre la salud. Aunque estas tres preocupaciones básicas coexisten en grado diverso según las distintas instituciones, la preocupación por el impacto de la educación médica sobre la salud emerge progresivamente como esencial.

El punto álgido en las Facultades de Medicina va a tener lugar en breve en cuanto se establezcan las directrices generales para el establecimiento de los curricula médicos ${ }^{7}$. Resulta imprescindible desde esta perspectiva establecer el concepto de diseño curricular, término amplio que en este análisis requiere necesariamente una definición, puesto que es en él donde encuentra sentido el segundo concepto de interés en este análisis: las competencias, que frecuentemente son consideradas aisladas del marco curricular, lo que genera dificultades para su comprensión y aplicación.

Asimismo resulta, no solo importante, sino necesaria la adecuada inserción de la Facultad de Medicina en la sociedad. ¿Cómo van a planificar las Facultades su acción para establecer vínculos con la sociedad, generando profesionales de calidad conforme a los requerimientos de salud de la población? La respuesta se encontrará en el "diseño curricular" de las Facultades, es decir, en los procesos de planificación de las acciones académicas.

\section{DISEÑO CURRICULAR}

El diseño curricular es mucho más que la definición del perfil profesional y la configuración de una malla secuencial y lógica de asignaturas y experiencias de aprendizaje organizadas para la formación de futuros médicos. Algunos autores ${ }^{8}$ definen el diseño curricular como una selección cultural y, como tal, un difícil ejercicio de "apreciar y excluir". Incluyendo una proyección de educación permanente, sabedores de que no será la única oportunidad de aprendizaje que tenga la persona. De esta manera, diseñar un currículo es tomar decisiones y nadie ha dicho nunca que esto sea fácil ${ }^{9}$.

El currículum debe responder a tres cuestiones ${ }^{10}$ :

A. ¿Cuál es el conocimiento válido? o ¿Qué deben aprender los estudiantes? Se trata de seleccionar y excluir contenidos, con el propósito de definir aquellos que son indispensables para la formación de los profesionales médicos. Acción esta de gran complejidad si se tienen en cuenta los veloces cambios que se generan actualmente en la esfera del conocimiento humano y en las crecientes demandas de la forma- 
ción profesional. Es aquí donde las competencias constituyen un modo de operar, una forma de articular educación y trabajo, Facultades de Medicina y Centros de Salud. Más allá de una moda, se trata del vínculo necesario del trabajo con la educación formal de los profesionales de la salud.

B. ¿Cómo adquieren los conocimientos, habilidades y actitudes? La segunda pregunta a la que debe responder el diseño curricular tiene que ver con las formas pedagógicas, o lo que se estima adecuado y coherente para la transmisión del conocimiento, aquí es donde adquieren importancia las ideas sobre cómo se aprende y cómo se enseña. Desde hace años, estos conceptos están siendo revisados y nuevos paradigmas plantean una serie de cambios en los enfoques, procesos y prácticas educativas. El aprendizaje se entiende ahora como un proceso de construcción individual mediante el cual se hace una interpretación personal y única de la cultura ${ }^{11}$. Desde esta perspectiva, los procesos de aprendizaje no son una mera asociación de estímulos y respuestas, o de acumulación de conocimientos, sino cambios cualitativos en las estructuras y esquemas existentes, de complejidad creciente. Aprender no consiste en hacer una copia o reproducción interna de la realidad o información externa, sino en hacer una interpretación y representación personal de dicha realidad $^{12}$.

El desarrollo de habilidades de orden superior, como las habilidades metacognitivas y la creatividad, son también fundamentales en un mundo en constante cambio e incertidumbre, donde cada día el profesional hace uso de una cantidad de información que demanda competencias relacionadas con el mejoramiento de sus habilidades de asociación, procesamiento y utilización de estrategias que potencien la capacidad de aprendizaje ${ }^{13}$. Por esto, las nuevas formas educativas para que puedan considerarse como tales, requieren no sólo de cambios estructurales, sino también de modificaciones en las prácticas educativas ${ }^{14}$. Lograr que docentes y alumnos participen de una manera más comprometida durante el proceso de enseñanza-aprendizaje será posible en la medida en que conozcan, interpreten y hagan suyas las nuevas propuestas curriculares enmarcadas en el modelo de las competencias profesionales integrales ${ }^{15}$.

C. ¿Cómo evaluar para el logro de las competencias y asegurar el dominio de ellas? La tercera pregunta del diseño curricular se refiere a cómo eva- luar el logro de los objetivos y experiencias de aprendizaje. Este es sin duda uno de los aspectos más complejos de la planificación educativa ${ }^{16}$. La función evaluadora tradicional se concibe como la congruencia entre la respuesta solicitada a los estudiantes y el objetivo de aprendizaje propuesto ${ }^{17}$. En este enfoque, para formar pensadores competentes, el docente tiene que concebir procedimientos y estrategias autorreguladoras del proceso de aprendizaje tanto para el estudiante como para el propio docente, que signifiquen un cambio del enfoque de la evaluación, refiriéndola en este caso a los criterios acordados previamente ${ }^{18}$.

\section{LAS COMPETENCIAS}

La competencia es una combinación integrada por conocimientos, habilidades y actitudes conducentes a un desempeño adecuado y oportuno de una tarea en el campo de las ciencias de la salud. El término competencia entonces puede ser definido de manera general como un "saber hacer sobre algo, con determinadas actitudes" ${ }^{19}$, es decir, como una medida de lo que una persona puede hacer bien como resultado de la integración de sus conocimientos, habilidades, actitudes y cualidades personales.

El término alude, en primer lugar, al carácter eminentemente práctico de toda competencia. Para saber si alguien es competente es indispensable observarle. No se es competente cuando sólo se sabe cómo "se debe hacer", sino cuando se hace efectivamente y de una manera adecuada. En segundo lugar, la definición hace referencia a algo sobre lo que se sabe hacer, que es el contenido de la competencia. En último lugar, para poder afirmar que alguien es competente no basta saber que hace ese algo, es muy importante la manera o la actitud con la que actúa. Nuevamente se hace referencia a los aprendizajes que hacen a alguien competente, saber que es, saber hacerlo, saber comunicarlo, saber como hacer para saberlo.

Según algunos autores ${ }^{9}$ pueden establecerse, de forma general, tres grupos de competencias:

En la base se encuentran las Competencias básicas, son aquellas con las que cada uno construimos nuestro aprendizaje. Están referidas fundamentalmente a la capacidad de "aprender a aprender", se elimina radicalmente la idea de que es posible aprender todo de una vez y para siempre, y de que en la Universidad se puede reproducir todo el conocimiento. Estas competencias requieren de habilidades básicas como: la capacidad para la expresión oral y escri- 
ta; movilizan rasgos cognitivos como son: la capacidad de comprender de manera crítica la información de diversas fuentes, la aptitud para observar, la voluntad de experimentación, la capacidad de tener criterio y de tomar decisiones $\operatorname{~etc}^{15}$. Entre las competencias básicas que suelen incluirse en los currícula se encuentran la comunicación verbal, la lectura y la escritura, el trabajo en equipo, la resolución de problemas, el dominio de lenguas extranjeras, etc ${ }^{19}$.

Otro grupo está constituido por las Competencias personales, se trata de aquellas que permiten realizar con éxito diferentes funciones en la vida: actuar responsablemente, mostrar deseo de superación y aceptar el cambio, entre otras.

Este grupo de competencias constituye un conjunto de difícil definición Ellas están en función de la capacidad y potencialidad de expresión de un grupo de características que se manifiestan en relación con el ambiente en que se desarrolle la actividad, por ejemplo: seguridad en sí mismo, capacidad para dominar los sentimientos y las tensiones emocionales, curiosidad, argumentación crítica y capacidad analítica.

Finalmente, el grupo de las Competencias profesionales. Estas son las que garantizan el cumplimiento de las tareas y responsabilidades del ejercicio profesional ${ }^{20}$. La capacidad de las personas para desenvolverse productivamente en una situación de trabajo no dependen sólo de las situaciones de aprendizaje académico formal, sino también, y de forma relevante, del aprendizaje derivado de la experiencia en situaciones concretas de trabajo ${ }^{15}$. Siempre se ha sabido "que uno estudia medicina, pero luego se hace médico en el ejercicio diario de la profesión". La integración de todas las competencias expuestas representan los factores críticos del éxito profesional.

\section{DISEÑO CURRICULAR BASADO EN COMPETENCIAS}

Los modelos actuales del diseño curricular se han redefinido para pasar de modelos centrados en la enseñanza a modelos centrados en el aprendizaje -estudiante- ${ }^{8}$. Estos curricula describen y proponen actividades que fortalezcan un aprendizaje duradero, transferible y autorregulable por el alumno. Teniendo en cuenta cómo percibe, codifica y elabora la información el sujeto; cómo la transforma a través de la experiencia en conocimiento y cómo la aplica para la solución de problemas y la generación de nuevos conocimientos.
En el diseño se establecen las competencias, se definen los objetivos de aprendizaje y las actividades a realizar, se formulan las metas y se describen los cursos. Estas acciones orientan la organización de los contenidos en unidades de aprendizaje y ayudan a seleccionar las estrategias para el planeamiento y diseño de las acciones. Aquí encuentran cabida metodologías como el aprendizaje basado en problemas (ABP), pacientes simulados, prácticas clínicas y otras centradas en el estudiante, en el desarrollo de sus procesos cognitivos superiores, de sus habilidades comunicativas y en la formación de actitudes.

En la evaluación, cuando el diseño curricular es por competencias y se quiere saber si alguien es competente, resulta indispensable observarle actuando; es decir, como ya se ha apuntado anteriormente, no se es competente cuando solo se sabe como se debe hacer, sino cuando se hace efectivamente y de una manera adecuada. Las modalidades de ECOE/OSCE y otras que se emplean en muchas Facultades de Medicina están orientadas en esta dirección ${ }^{21}$. El problema de las instituciones de educación superior sería establecer ¿Cuándo se certifica que el alumno ha logrado el dominio de las competencias?

Para ello, las Facultades de Medicina tienen como tarea fundamental la identificación de los componentes básicos del proceso educativo ${ }^{22}$, es decir, la respuesta a los interrogantes iniciales de este comentario: ¿Qué deben aprender los estudiantes? ¿Cómo adquieren los conocimientos, habilidades y actitudes? y ¿Cómo evaluar el logro de las competencias y asegurar el dominio de ellas?

\section{CONSIDERACIONES FINALES}

El modelo educativo por competencias profesionales integradas para la educación médica es una opción que busca generar procesos formativos de mayor calidad, pero sin perder de vista las necesidades de la sociedad, de la profesión, del desarrollo de la profesión y del trabajo académico. Asumir esta responsabilidad implica que las Facultades de Medicina no sólo redefinan sus proyectos educativos, sino que promuevan de manera congruente acciones en los ámbitos pedagógicos que se traduzcan en modificaciones reales de las prácticas docentes ${ }^{23}$. De ahí la importancia de que el docente también participe de manera continua en las acciones de formación y capacitación que le permitan desarrollar competencias similares a aquellas que se busca formar en los estudiantes.

La incorporación del modelo curricular por competencias significa, además, el establecimiento y 
consolidación de profundas relaciones entre las Facultades de Medicina, los egresados y los centros de salud en que desarrollan su trabajo profesional, como única forma de garantizar una interacción que permita definir en caso necesario, las modificaciones pertinentes en el diseño curricular que aseguren el desarrollo de las competencias requeridas en cada caso.

El equilibrio necesario entre los factores implicados y las condiciones y necesidades del mundo actual, cada vez más velozmente cambiantes, convierten esta labor en un presupuesto teórico de difícil resolución práctica ${ }^{24}$. Por esto el modelo de desarrollo curricular por competencias supone un acercamiento más dinámico a la realidad del mundo circundante, pero que sólo puede ser acometido desde una visión integral del papel del docente y si el estudiante asume un papel activo en su aprendizaje.

\section{RECOMENDACIÓN}

Este grupo de docentes (FE-FAU), preocupados por la adecuada y veloz adaptación de las Universidades a la nueva época asume como referente en el ámbito de las competencias médicas el trabajo realizado por diferentes comités del Instituto para la Educación Médica Internacional (creado en 1999 por la "China Medical Board of New $\underline{\text { York }})^{25,26}$. Dicho Instituto ha definido las "competencias esenciales mínimas" que todo licenciado en Medicina debería poseer para ser reconocidos como un "médico trasnacional" (o globalizado, ${ }^{25}$ ), de forma que quienes las posean podrían seguir la formación postgraduada en una especialidad médica en cualquier lugar del mundo. Asimismo recomienda las acciones realizadas por el grupo de $\mathrm{Harden}^{27}$ para Escocia y, en nuestro entorno, al proyecto DIS$S E N Y^{28}$ de Cataluña. Respecto a la formación del profesorado, anima, estimula y recomienda la realización de programas de formación avanzada en Educación Médica (v.g. maestría de la Universidad de Chile, master de la Universidad de Castilla-La Mancha, etc) y presta su respaldo y total apoyo, a todas y cada una de las diferentes acciones que en la andadura hacia el EEES se están realizando en diferentes Facultades. En esta línea insta a todos, órganos de gobierno académico, sociedades profesionales y foros independientes de opinión a proseguir en el camino no regateando esfuerzos en el apasionante reto de la Refundación de la Universidad en este cambio de época en los comienzos del siglo XXI.

\section{AGRADECIMIENTOS}

Los trabajos de este grupo han sido financiados por el programa de Redes Temáticas de la Agencia Española de Cooperación Internacional (M.A.E.C.) y por las universidades participantes.

\section{BIBLIOGRAFÍA}

1. González Ramírez T. El Espacio Europeo de Educación Superior: Una nueva oportunidad para la Universidad. En: La Universidad en la Unión Europea. El Espacio Europeo de Educación Superior y su impacto en la docencia, (eds: Colás MP y de Pablos, J) Ediciones Aljibe, Málaga, 2005; pp 27-55

2. Colás MP y de Pablos J. Introducción. En: La Universidad en la Unión Europea. El Espacio Europeo de Educación Superior y su impacto en la docencia. Málaga: Aljibe, 2005; pp 15-25

3. Tiana A. Prólogo. En: La Universidad en la Unión Europea. El Espacio Europeo de Educación Superior y su impacto en la docencia (eds: Colás MP y de Pablos, J). Málaga: Aljibe, 2005; pp 11-13

4. Colás MP. La formación universitaria en base a competencias. En: La Universidad en la Unión Europea. El Espacio Europeo de Educación Superior y su impacto en la docencia (eds: Colás MP y de Pablos, J). Málaga: Aljibe, 2005; pp 101-123

5. Palés J. et al. Defining the learning outcomes of graduates from the medical school at the University of Barcelona. (Catalonia, Spain). Medical Teacher 2004; 26 (2): 1-5

6. Boelen C. Global Challenges in Medical Education. En: Libro de Actas del I Congreso de la SEMDE. San Sebastián: SEMDE, 2004; pp 7-8

7. Palés J. La educación médica basada en las competencias finales del estudiante. Educ Med 2001; 4: 1

8. Sacristán JG. El currículum: Una reflexión sobre la práctica. Madrid: Morata, 1998.

9. Huerta J, Pérez S, Castellanos AR. Desarrollo Curricular por Competencias Profesionales Integrales. México: Educar, 2000.

10. Castillo M. Perfil Docente de los académicos de la Facultad de Medicina [Tesina]. Santiago de Chile: Universidad de Chile, 2003.

11. Coll C, Martín E, Mauri T, Miras M, Onrubia J, Solé I, et al. El constructivismo en el aula. Buenos Aires: Prometeo, 2002.

12. Ausubel D. Psicología Educativa: Un punto de vista cognoscitivo. México: Trillas, 1983.

13 Alonso CM, Gallego D.J., y Honey P. Los estilos de aprendizaje. Procedimientos de diagnóstico y mejora. Bilbao: Ediciones Mensajero, 2002.

14. Barell J. El Aprendizaje basado en problemas. Buenos Aires. Manantial, 1999.

15. Gonzki A. Instrumentación de la educación basada en competencias. En: Competencia laboral y educación basada en normas de competencia. México: Limusa, 1996.

16. Torres J. El curriculum oculto. Madrid: Morata, 1998.

17. Himmel E. La evaluación escolar. Santiago de Chile: Editorial Universitaria, 1972.

18. Flores R. Evaluación pedagógica y cognición. México: Mc Graw Hill, 2000.

19 Irigoin M, F.Vargas. La formación basada en competencias. En: 
Competencia laboral; manual de conceptos, métodos y aplicaciones en el sector salud. Montevideo: OPS C, 2002; 177-214

20. Nolla M, Palés J, Gual A. Desarrollo de las competencias profesionales. Educ Med 2002; 5: 76-81

21. Sociedad Española de Educación Médica (SEDEM). Declaración del Lazareto de Mahon: Evaluación de las competencias profesionales en el pregrado. Educación Médica 2004; 7: 103-105.

22. Gutiérrez J.A., Millán J. y Villanueva J.L. Introducción a "Competencias médicas: desde la Facultad de Medicina hasta la especialización médica”. Educ Med 2005; 8 (supl 2): 3

23. Martínez F. El perfil del profesor universitario en los albores del siglo XXI. Primer encuentro de perfeccionamiento del profesorado. U. de Murcia. España: http://edutec.rediris.es/documentos/1999/perfil.htm (visitado Abril 2006)

24. Magendzo A. Pedagogía crítica y educación en derechos humanos. Paulo Freire, Revista de Pedagogía Crítica. 2003; 2 (2):19-27

25. Schwarz, M.R. y Wojczak, A. Una vía hacia la educación médica orientada a las competencias: los requisitos globales esenciales mínimos. Educ Med 2003; 6 (S 2): 5-10 (traducido del "Medical Teacher 2002; 24: 125-129 por Albert Oriol-Bosch)

26. Comité Central del Instituto para la Educación Médica Internacional. Requisitos globales mínimos esenciales en educación médica. Educ Med 2003; 6 (S 2): 11-19 (traducido del Medical Teacher 2002; 24 (2): 130-155 por Juan Mendoza Vega)

27. Harden, R.M., Crosby, J. R., Davis, M.H. and Friedman, M. From competency to meta-competency: a model for the specification of learning outcomes. AMEE Education Guide 14: 37-45, 1999.

28. Carreras, J. Competencias básicas comunes de los Licenciados en Medicina de las Universidades Catalanas: programa "Disseny". Educ Med 2005; 8 (3): 137

\title{
*RESEÑA DE LA RED: \\ RED IBEROAMERICANA PARA LA FORMACIÓN CONTINUA DE LOS DOCENTES DE AREAS BIOMÉDICAS. "FORMAS DE ENSEÑAR - FORMAS DE APRENDER EN LA UNIVERSIDAD" (FE-FAU)
}

\author{
Gabriela Díaz-Veliz ${ }^{4}$, Pascual Gargiulo ${ }^{5}$, Ricardo Bianchi ${ }^{5}$, Dorian Gorena $^{6}$ y José Mayora ${ }^{6}$ \\ 1 (U. del País Vasco-Euskal Herriko U., Leioa, España, coordinador); \\ 2 (U. de Zaragoza, Zaragoza, España); \\ 3 (U. de Valladolid, Valladolid, España); \\ 4 (U. de Chile, Santiago de Chile, Chile); \\ 5 (U. Ncal. de Cuyo, Mendoza, Argentina); \\ 6 (U. Andina Simón Bolivar y U. Mayor de S. Fco. de Chuquisaca, Sucre, Bolivia).
}

José-Vicente Lafuente ${ }^{1}$, Jesús F. Escanero ${ }^{2}$, Jose $M^{a}$ Manso $^{3}$, Sergio Mora ${ }^{4}$, Teresa Miranda ${ }^{4}$, Manuel Castillo ${ }^{4}$,

Esta red se constituyó en 2003 auspiciada por la Agencia Española de Cooperación Internacional (AECIMAEC) configurando un foro de análisis e intercambio de experiencias sobre la formación continua de los docente universitarios en el área de las ciencias biomédicas, tratando de dinamizar y armonizar dicha formación, en lo que se refiere a sus competencias docentes.

Las actividades de la Red se desarrollan a través de la participación en congresos y de la realización de symposia propios abiertos a todos los interesados.

Las acciones en este campo reportan evidentes beneficios para los usuarios últimos, que son directamente los estudiantes de estas carreras e indirectamente el sistema de salud y por ende la sociedad en su conjunto. El enorme desarrollo experimentado por las ciencias biomédicas demanda adecuar y priorizar los contenidos del curriculum según el contexto social en que se van a desarrollar, así como actualizar las competencias docentes del profesorado. En este contexto parece oportuno que esta actualización de la capacitación de los docentes se lleve a cabo en un marco plural como el que generan redes de esta índole. 